\title{
DEVELOPMENTAL ENVIRONMENT ALTERS CONDITIONAL AGGRESSION IN ZEBRAFISH
}

\author{
A Thesis \\ Presented to \\ The Graduate Faculty of The University of Akron \\ In Partial Fulfillment \\ of the Requirements for the Degree \\ Master of Science
}

Chris Marks

August, 2006 


\title{
DEVELOPMENTAL ENVIRONMENT ALTERS CONDITIONAL AGGRESSION IN
}

\section{ZEBRAFISH}

\author{
Chris Marks
}

Thesis

Approved:

Advisor

Dr. Francisco Moore

Committee Member

Dr. Stephen C. Weeks

Committee Member

Dr. Brian Bagatto

Department Chair

Dr. Bruce Cushing
Accepted:

Dean of College

Dr. Ronald F. Levant

Dean of the Graduate School

Dr. George R. Newkome

Date 


\begin{abstract}
The developmental environment influences a wide variety of phenotypic traits in the adults of many vertebrates (i.e., developmental plasticity). In this study, we test to see if developmental environment $\left(\mathrm{E}_{\mathrm{DEV}}\right)$ interacts with the adult behavioral environment ( $\left.\mathrm{E}_{\mathrm{BEHAV}}\right)$ in determining behavioral phenotypes. We reared Zebrafish (Danio rerio) from eggs in either continuously hypoxic or normoxic conditions. We then tested aggression and avoidance (i.e., hiding) levels of fish from each developmental treatment in both environments. Developmental environment was a significant source of variation in avoidance behavior while the stimulus environment did not influence avoidance. Without a period of acclimation we found that $E_{\mathrm{BEHAV}}$ and an $\mathrm{E}_{\mathrm{DEV}} \mathrm{X} \mathrm{E}_{\mathrm{BEHAV}}$ interaction were both significant sources of variation. However, when the fish were allowed to physiologically acclimate to the environment for $16 \mathrm{~h}$, aggression level was highest for fish tested in the environment in which they developed. In that case the $\mathrm{E}_{\mathrm{DEV}} \mathrm{X} \mathrm{E}_{\mathrm{BEHAV}}$ interaction was the only significant source of variation. These results demonstrate that a more complete understanding of phenotypic response can be gained by incorporating environmental conditions across multiple time scales.
\end{abstract}




\section{ACKNOWLEDGEMENTS}

We thank R. Wani for assistance in the lab. This research was funded in part by a University of Akron Department of Biology Undergraduate Research Grant to T. West and the Muehlstein Academy in Mathematics and Science. Animals used in this research were handled in accordance with University of Akron IACUC protocol \#02-10-A. 
TABLE OF CONTENTS

Page

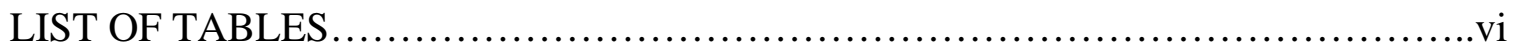

LIST OF FIGURES.............................................................

\section{CHAPTER}

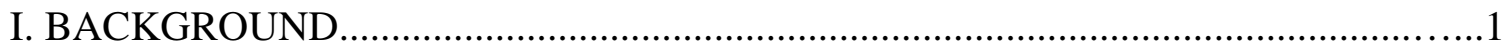

II. MATERIALS AND METHODS....................................................

Stock and Developmental Conditions.......................................4

Experimental Designs..................................................6

Behavioral Assays............................................................

Statistical Methods....................................................... 8

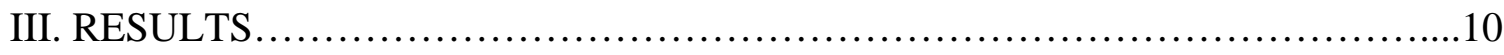

No Acclimation Experiment..............................................10

16-h Acclimation Experiment................................................10

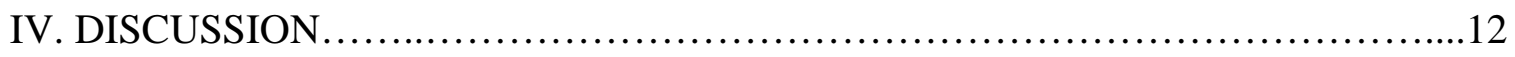

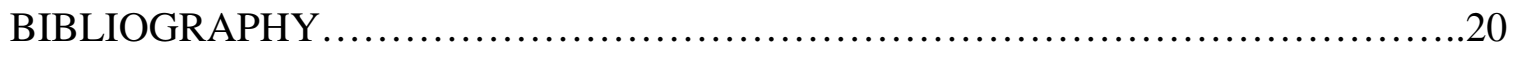




\section{LIST OF TABLES}

Table

Page

1. Aggression analysis.................................................... 15

2. Avoidance analysis..................................................... 16 


\section{LIST OF FIGURES}

Figure Page

1. Hypothetical reaction norms...................................................

2. Aggression results......................................................... 18

3. Avoidance results............................................................... 


\section{CHAPTER I}

\section{BACKGROUND}

Classic quantitative genetic models divide the fundamental factors influencing phenotypic variation into genetic and environmental (Falconer, 1981; Pigliucci, 2001; Dewitt and Scheiner, 2004b). Both of these factors as well as an interaction between the two can be important contributors to variation in a given trait. The genetic sources of variation can be further divided into additive and non-additive genetic components (Falconer, 1981). Additive genetic variation is the result of the independent action of genes on trait value. Non-additive genetic variation is created primarily by genetic interactions. While gene-gene and gene-environment interactions are increasingly recognized as important in evolution (Whitlock et al., 1995; Scheiner and Dewitt, 2004), interactions between ontogenetic (developmental) environments have largely been ignored and are also potentially important evolutionary influences (Pigliucci, 2001; Sih, 2004). In this study, we test for additive and interaction effects of developmental and adult environments in Zebrafish (Danio rerio) behavior.

Environmental influence on traits (plasticity) can occur at any level of organismal hierarchy including biochemistry, physiology, morphology, and behavior (Pigliucci, 2001; Schlichting and Smith, 2002; Sih, 2004). The timescale of the plastic response varies from acute (Brenowitz et al., 1998; Sih, 2004) to broadly ontogenetic (West- 
Eberhard, 2003). Adaptive evolution of a plastic phenotype requires genetic variation for the plastic response or genotype by environment (G X E) interactions in the production of phenotypic variation. Plasticity and G x E interactions are well studied (Via and Lande, 1985; Stearns, 1989; West-Eberhard, 2003), with hundreds of studies finding variation in plasticity on which selection can act (Newman, 1992; Pigliucci, 2001; Dewitt and Scheiner, 2004a).

Variation in environment over the ontogeny of an organism creates numerous inputs into trait variation. Developmental plasticity, in which juvenile environment molds adult phenotype, is common (West-Eberhard, 2003) and may be beneficial if juvenile environment predicts adult environment. Shorter time-scale plastic effects, such as physiological acclimatization or context-sensitive behavior, are ubiquitous (Pigliucci, 2001; Sih, 2004) and likely to be more useful when the animal experiences unpredictable changes throughout its ontogeny. Although the combined influence of environmental effects across these scales has been largely ignored, it has recently been argued that current optimality models may fail as a consequence of conflicts between plasticity at different scales (Sih, 2004).

Environmental effects on traits occurring at different time scales may be purely additive, or acute plasticity may be contingent on the developmental environment. Analogous to genetic factors, environments experienced sequentially during an animal's ontogeny combine to influence a phenotype, and their effects can be decomposed into additive and interaction sources of variation. Environmental interactions are a potentially critical and understudied source of phenotypic variation. Figure 1 provides a set of hypothetical phenotypic effects that result from ontogenetically distinct environments and 
their interactions. It is presented in a typical format for genotype and environment effects (Via and Lande, 1987), but in this case, different reaction norms represent differing developmental environments rather than differing genotypes. Interactions of the type in Figure 1C should be represented by environment by environment (E X E) interactions in a traditional quantitative genetic framework. Explicit measures of this type of environmental interaction in the production of phenotype are unexplored in quantitative genetics.

In this study, we differentiate between the developmental environment and the acute behavioral environment as causes of aggression and avoidance behavior in Zebrafish. We tested for plastic response by rearing laboratory populations of Zebrafish from egg to adulthood in normoxic and hypoxic conditions. We then measured aggression and avoidance levels in fish from both developmental treatments in both environments. We further tested whether plastic responses declined during a period of physiological adjustment (acclimation). 


\section{CHAPTER II}

\section{MATERIALS AND METHODS}

We chose Zebrafish as a focal species and oxygen concentration as our environmental variable for the following reasons. Hypoxia presents a common stress in many fish species, producing a suite of organismal, cellular, and genetic responses (Gracy et al., 2001; Nikinmaa, 2002; Ton et al., 2003). Hypoxic stress in fish can be acute, seasonal, or chronic and hypoxia has been demonstrated to cause developmental delays (Rombough, 1988) and alter the regulation of over 100 genes (Gracy et al., 2001; Ton et al., 2003) in several fish species. Plastic response of fish to hypoxia stress has been demonstrated to produce ecologically relevant morphological tradeoffs (Chapman et al., 2000). Finally, because Zebrafish are found across a wide variety of habitats from fast-moving rivers to slow moving stagnant bodies of water (Talwar and Jhingran, 1991), they are a good candidate to display complex plastic behavior in response to oxygen level.

Stocks and Developmental Conditions

Eggs used in the experiment were the product of individual mating of parents from three different sources: wild-caught fish from India, commercially bred fish with a history of regular backcrossing to wild fish, and relatively inbred commercial laboratory strains (trade name “'Longfin Gold”). Wild stocks were obtained through Poseidon 
Aquatics (Gardena, CA), and both commercial strains were obtained from Scientific Hatcheries Inc. (Huntington Beach, CA). An assortment of crosses between and within the available stocks produced 631 eggs from 12 full-sib clutches. Each clutch was split with an equal number of eggs placed in each environment. For logistical reasons all fish within a developmental environment were pooled as the individual fish matured (40 days). Genetic variation could therefore not be partitioned within environments. The genetic variation present was, however, balanced across the two developmental environments and randomized across behavioral environments. This allowed testing for the average plastic response across a diversity of genotypes. The average survivorship to day 40 across clutches was not significantly different between oxygen treatments (paired t-test $\mathrm{P}=0.390$ ) with normoxic and hypoxic survivorship being $69 \%$ and $64 \%$, respectively.

Within two hours post-fertilization, eggs were placed in their developmental environment. A split sump system of 20 tanks (total volume approx. 1200 l) allowed passive and active exchange of water between the normoxic and hypoxic tanks. Normoxic tanks were fed with water that was continuously oxygenated to near saturation (DO approx. $6.8 \mathrm{mg} / \mathrm{l}$ ) using airstone bubblers on the normoxic side of the sump. The hypoxic tank’s DO concentration was maintained at $0.8 \mathrm{mg} / \mathrm{l}(+/-0.1 \mathrm{mg} / \mathrm{l})$ by sparging with N2 in a series of 2 m tall sparging columns controlled by a YSI 5200 monitoring system. Light was provided on a 14L : 10D cycle using built in control systems in the rearing rooms. A push-pull heating and chilling system maintained the temperature at 27 +/- 0.5 C. All fish were fed twice daily on a commercial diet obtained from Scientific 
Hatcheries Inc. The fish were raised in those conditions to adulthood (roughly 75 days) when they were assayed.

Experimental Designs

Two aggression experiments were carried out using the split clutches. In the first of these experiments (no acclimation experiment), 128 fish from each developmental environment were assayed in two complete blocks (64 fish per block). Each fish was taken from its developmental environment and placed in an environmental chamber that contained either hypoxic or normoxic water. The fish was then given a 10-min behavioral adjustment (accommodation) period in a test arena. After accommodation, the fish's behavior was videotaped for two min in order to determine the aggressiveness of the fish. Half of the fish were tested in their own developmental environment and the other half were tested in the opposite environment (i.e., there were no repeated measures).

The second experiment (16-h acclimation experiment) was identical to the first except that a new sample of fish from each developmental environment was used (one block of 64 fish) and the fish were placed into a holding tank in the oxygen environment that they would be assayed in for $16 \mathrm{~h}$ prior to being placed into the behavioral chamber. This allowed animals to acclimate to the oxygen environment that they would experience in the assay. After this acclimation period, they were moved into the assay chamber and given a 10-min accommodation period before being videotaped for two min as in the first experiment.

\section{Behavioral Assays}

The aggression assays were performed in $30 \times 30 \times 30 \mathrm{~cm}$ open top opaque white 
polyethylene tank. The opacity of the material helped ensure that the fish were secluded from outside disturbances. The tank was filled to roughly $20 \mathrm{~cm}$ depth and supplied with a continuous flow of water from the appropriate sump for their treatment. The flow through rate was adjusted so that there was no visible effect of currents on fish swimming in the immediate vicinity (approx. $1 \mathrm{~cm}$ ) of the inflow or drain, allowing the fish to swim about the tank freely. Dissolved oxygen for the behavioral environments were not detectably different from the equivalent rearing environment and shared the same chemical composition and temperature as the rearing environments.

A mirror image of the subject provided a stimulus for aggressive behavior. Mirror image stimulation (MIS) is a well-established method for studying fish behavior (Tinbergen, 1951). Mirror image stimulation has the unique benefit of providing immediate feedback to the subject's activity and alleviates the need for invasive procedures and excess handling (Rowland, 1999). A $10.16 \mathrm{~cm}$ high by $7.6 \mathrm{~cm}$ wide stainless steel mirror was suspended below the waterline against the center of one wall of the behavioral chamber. The time spent (in seconds) butting or nipping the mirror was recorded as aggressive behavior when the tapes were reviewed. In normal stocking densities, we have not noticed any schooling behavior of Zebrafish, while chases and fin nipping are common. In pilot two-way behavioral experiments (West, unpubl. data), such direct contact also always resulted in either fin nipping or forceful collisions. Given that these interactions were likely to cause damage to one or both fish, we felt confident that direct contact of a fish with its mirror image could conservatively be construed as aggressive behavior rather than a schooling response.

A plastic aquarium plant was placed near the opposite end of the tank from the 
mirror in order to provide a hiding place in which fish could take refuge from their own reflection. The time (in seconds) spent hiding in the foliage was used as a measure of avoidance behavior. We use the term avoidance in general and not specifically as an avoidance of the mirror image. Fish were only counted as hiding if they pushed into the foliage (i.e., utilized the cover) and remained stationary in that cover. General preference for the vegetation, when fish frequented the cover but did not utilize it, was not recorded as avoidance. Immediately after each assay was complete, the wet weight of that fish was recorded.

\section{Statistical Methods}

Developmental environment, behavioral environment (i.e., acute test environment), and their interaction were each of interest as possible sources of variance in both experiments. An ANCOVA model was used to test for developmental environment $\left(\mathrm{E}_{\mathrm{DEV}}\right)$, behavioral environment $\left(\mathrm{E}_{\mathrm{BEHAV}}\right)$, and an EDEV by $\mathrm{E}_{\mathrm{BEHAV}}$ interaction ( $\left.\mathrm{E}_{\mathrm{DEV}} \mathrm{x} \mathrm{E}_{\mathrm{BEHAV}}\right)$ as sources of variation in aggression and avoidance behavior. The across-treatment influence of wet weight was statistically removed since fish size might have influenced levels of aggression. Analyses were performed as ANCOVAs in the SAS GLM procedure (SAS Institute Inc., Cary, NC) with wet weight treated as a covariate. A t-test was performed to test for differences between the developmental treatments in wet weight. Block was included as a source of variation in the analysis of the no acclimation experiment while the 16-h acclimation experiment was completed in a single block. Since the average time spent in aggression and hiding is relatively low, a Poisson rather than normal distribution of residuals was expected in the data (Sneedecor 
and Cochran, 1989). We therefore applied square root transformations to both response variables to improve the normality of the residuals. 


\section{CHAPTER III}

\section{RESULTS}

\section{No Acclimation Experiment}

$\mathrm{E}_{\mathrm{DEV}}$ was a significant source of variation in aggression (Table 1a) and avoidance (Table 2a). The average aggression of hypoxia-reared fish was lower than that of normoxia-reared fish (Fig. 2a), and hypoxia-reared fish spent more time in hiding than did normoxia-reared fish (Fig. 3a). EBEHAV did not contribute to the variation in aggression except through a significant $E_{\mathrm{DEV}} \mathrm{x} \mathrm{E}_{\mathrm{BEHAV}}$ interaction. Fish were, on average, more aggressive in their own developmental environment (Fig. 2a). EBEHAv did not affect avoidance behavior (Fig. 3a) either directly or through an $E_{\mathrm{DEV}} \mathrm{x} \mathrm{E}_{\mathrm{BEHAV}}$ interaction (Table 2a).

Wet weight added significantly to the variation in aggression (Table 1a) with larger fish being more aggressive. Wet weight was also a significant source of variation in avoidance behavior (Table 2a), with larger fish spending less time on avoidance. A ttest showed no significant difference in wet weight between the two developmental environments for this experiment $(\mathrm{P}>0.773)$.

\section{6-h Acclimation Experiment}

Neither $E_{\text {DEV }}$ nor $E_{\text {BEHAV }}$ were significant sources of variation in aggression except through an $E_{\mathrm{DEV}} \mathrm{X} \mathrm{E}_{\mathrm{BEHAV}}$ interaction (Table 1b). In hypoxia, hypoxia-reared fish 
were most aggressive, while in normoxia, normoxia-reared fish were most aggressive (Fig. 2b). Only EDEv was a significant source of variation in avoidance behavior (Table 2b). Across both environments, hypoxia reared fish again spent more time hiding in the vegetation (Fig. 3b).

Wet weight was not a significant source of variation in aggression (Table 1b) or avoidance behavior (Table 2b). A t-test showed no significant difference in wet weight between the two developmental environments for this experiment $(\mathrm{P}>0.09)$. We ran the model both with and without wet weight as a covariate and again found no qualitative changes resulting from the inclusion of wet weight. 


\section{CHAPTER IV \\ DISCUSSION}

Hypoxic stress during development clearly affects the behavioral response of Zebrafish. However, the response is not simply an increase or reduction in aggressive behavior. Instead, the effect of developmental environment on aggression is conditional on the behavioral environment (Table 1). Fish are most aggressive in their native environment (Fig. 2). In Zebrafish, which experience a broad range of environments, (Talwar and Jhingran, 1991), this may affect the ecological and evolutionary dynamics of the species.

It is likely that aggressive behavior is energetically costly for a fish and this cost may be exacerbated when fish are moved to a novel oxygen environment. In this experiment, normoxia-reared fish were just as aggressive as hypoxia-reared fish when exposed to the hypoxia for less than 15 min (Fig. 2A). However, when they were acclimated to hypoxia for $16 \mathrm{~h}$, they did not maintain that high level of aggression and were less aggressive than hypoxia-reared fish (Fig. 2B). This pattern suggests that while in this case developmental plasticity may be adaptive, behavioral plasticity may be partially due to nonadaptive constraints. It appears that in hypoxic water, normoxiareared fish may be incapable of maintaining costly behaviors and vice versa. Zebrafish reared in normoxia and placed in hypoxic water produce significantly higher lactic acid 
concentrations compared to hypoxia-reared Zebrafish (Widmer, Moore, and Bagatto, unpubl. data) indicating that oxygen may limit behavioral dynamics. The pattern of environmentally contingent behavior we found is in keeping with a correlated behavioral response to developmental changes in physiological or morphological traits that confer an advantage in a constant habitat.

Avoidance behavior in the presence of a mirror image stimulus was not contingent on the behavioral environment (Table 2). Across both behavioral environments, hypoxia-reared fish spent more time hiding than normoxia-reared fish and neither group of fish substantially altered their hiding behavior in response to behavioral environment. In short, for avoidance there was no behavioral plasticity. Developmental plasticity leading to avoidance behavior in hypoxia-reared fish could be either an adaptive “energy saving”' strategy or a non-adaptive consequence of a lack of available resources for more energetically costly pursuits.

The adaptive benefit of plasticity of aggression and avoidance behaviors is not fully resolvable within this study. There is, however, ample reason to think that such behavior may influence the dynamics of Zebrafish populations. Developmentally plastic organisms expressing unique phenotypes under the influence of a novel environment could allow invasion of that habitat, facilitating further adaptation. Ontogenetic factors clearly influence behaviors that may be critical in determining the success of Zebrafish entering novel oxygen regimes. These changes result in fish that are more timid in novel oxygen environments. This could alter the rates of adaptation to a changing habitat, range expansion or contraction, and competition as well as other evolutionary and ecological processes. Convincing arguments have been made for plasticity as an agent of 
micro- and macroevolutionary change in general (Via and Lande, 1985; Agrawal, 2001; West-Eberhard, 2003). Plastic responses have been recognized as being necessary for colonization of novel habitats (West-Eberhard, 1989; Agrawal, 2001), and plasticity in this case could allow invasion of novel habitats if lowered aggression is optimal in those habitats. This particular pattern of plastic response could also be an impediment to invasion of, and adaptation to, a novel environment if aggressive behavior is lowered below its optimum level in that novel environment.

We found that EDEv $\mathrm{X} \mathrm{E}_{\mathrm{BEHAV}}$ interactions are a critical source of variation in aggressive behavior in Zebrafish. The interaction between the developmental and adult environments is relatively poorly understood from an evolutionary perspective. There are, nevertheless, certain areas in which these types of interactions may be common. Specifically, learned and conditioned responses represent a case where varied experience earlier in life dictates the behavior of individuals later in life (Sih, 2004). For instance, in the squid, Loligo opalescens, exposure to copepods during early development determines prey capture behavior during later developmental stages (Chen et al., 1996). In such cases, prediction of an individual's behavior requires knowledge of both the current environment and previous environments.

In this study, we have looked at the interaction between the developmental environment and the adult environment in the creation of behavioral phenotypes. We introduce E X E interactions as a novel means of quantifying the integrated influence of ontogenetic environments. Sih (2004) has recently pointed out the potential importance of such joint developmental and behavioral responses in shaping evolutionary change, although in a different heuristic context. In general, when E X E interactions affect 
phenotypic variation in any type of trait, we must account for those interactions if we are to understand the evolutionary dynamics of those traits.

In summary, Zebrafish display plasticity in both aggression and avoidance across oxygen environments. In the case of aggression, it is the interaction of developmental environment and the behavioral environment that determines a fish's responses. In the case of avoidance, only the developmental environment influences the behavior. The existence of complex plasticity in aggressive behavior across oxygen environments indicates that more study of the adaptive significance of this particular trait is necessary. We conclude that a simple expansion of current studies to detect E X E interactions may elucidate the extent to which multiple environments seen during ontogeny combine to determine phenotype. 
Table 1: Aggression analysis. Analysis of covariance for time spent on aggressive behavior (A) without and (B) with acclimation. Sources of variation included developmental environment (EDEv), behavioral environment ( $E_{\text {BEHAV }}$ ), the interaction between developmental and behavioral environments (EDEv X E weight was included in the analysis as a continuous covariate.

\begin{tabular}{lcccc}
\hline \multicolumn{1}{c}{ Source } & DF & M S & F & P \\
\hline Wet weight & 1 & 34.765 & 6.84 & 0.0100 \\
A) Without Acclimation & & & & \\
Block & 1 & 41.321 & 8.13 & 0.0051 \\
E $_{\text {DEV }}$ & 1 & 42.220 & 8.31 & 0.0047 \\
E $_{\text {BEHAV }}$ & 1 & 0.432 & 0.09 & 0.7711 \\
E $_{\text {DEV X E EEHAV }}$ & 1 & 34.237 & 6.74 & 0.0106 \\
Error & 122 & 5.080 & & \\
& & & & \\
B) With Acclimation & & & & \\
Wet weight & 1 & 0.399 & 0.100 & 0.7566 \\
E $_{\text {DEV }}$ & 1 & $<0.000$ & $<0.000$ & 0.9996 \\
E $_{\text {BEHAV }}$ & 1 & 5.956 & 1.450 & 0.2337 \\
E $_{\text {DEV X E EHAV }}$ & 1 & 29.290 & 7.120 & 0.0098 \\
Error & 59 & 4.115 & & \\
\hline
\end{tabular}


Table 2: Avoidance analysis. Analysis of covariance for time spent on avoidance behavior (A) without and (B) with acclimation. Sources of variation included developmental environment $\left(E_{\mathrm{DEV}}\right)$, behavioral environment ( $\left.\mathrm{E}_{\mathrm{BEHAV}}\right)$, the interaction between developmental and behavioral environments ( $\left.E_{\mathrm{DEV}} \mathrm{X} \mathrm{E}_{\mathrm{BEHAV}}\right)$ and Block. Wet weight was included in the analysis as a continuous covariate.

\begin{tabular}{lcccc}
\hline \multicolumn{1}{c}{ Source } & DF & M S & F & P \\
\hline Wet weight & 1 & 23.647 & 4.97 & 0.0276 \\
A) Without Acclimation & & & & \\
Block & 1 & 2.647 & 0.56 & 0.4570 \\
E DEV $_{\text {BEHAV }}$ & 1 & 58.985 & 12.40 & 0.0006 \\
E $_{\text {DEV }}$ X E EEHAV & 1 & 4.612 & 0.97 & 0.3266 \\
Error & 1 & 1.395 & 0.41 & 0.5247 \\
& 122 & 4.755 & & \\
B) With Acclimation & & & & \\
Wet weight & 1 & 6.883 & 0.87 & 0.3588 \\
E $_{\text {DEV }}$ & 1 & 39.544 & 4.98 & 0.0295 \\
$E_{\text {BEHAV }}$ & 1 & 7.167 & 0.90 & 0.3461 \\
$E_{\text {DEV X E EEHAV }}$ & 1 & 1.107 & 0.14 & 0.7102 \\
Error & 59 & 7.946 & & \\
\hline
\end{tabular}



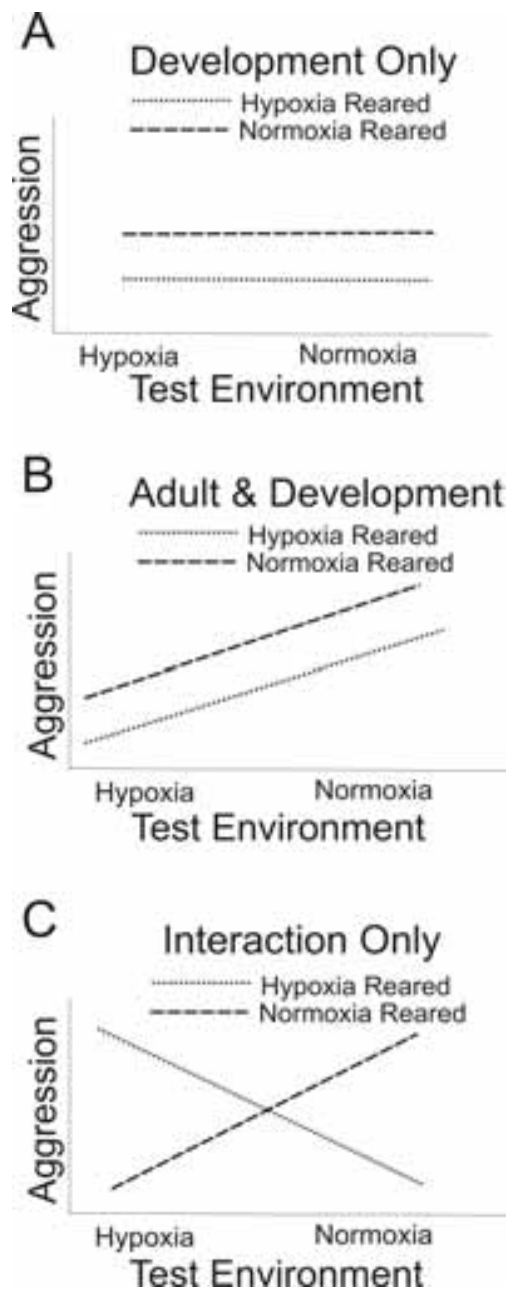

Figure 1: Hypothetical norms of reaction representing plastic response to (A) developmental environment only, (B) acute and developmental environment acting additively, and (C) an interaction where developmental environment influences aggression only by reversing the effect of acute environment or vice versa. The solid line represents the response of normoxia-reared individuals across environments. The dashed line represents the response of hypoxia-reared individuals across environments. 

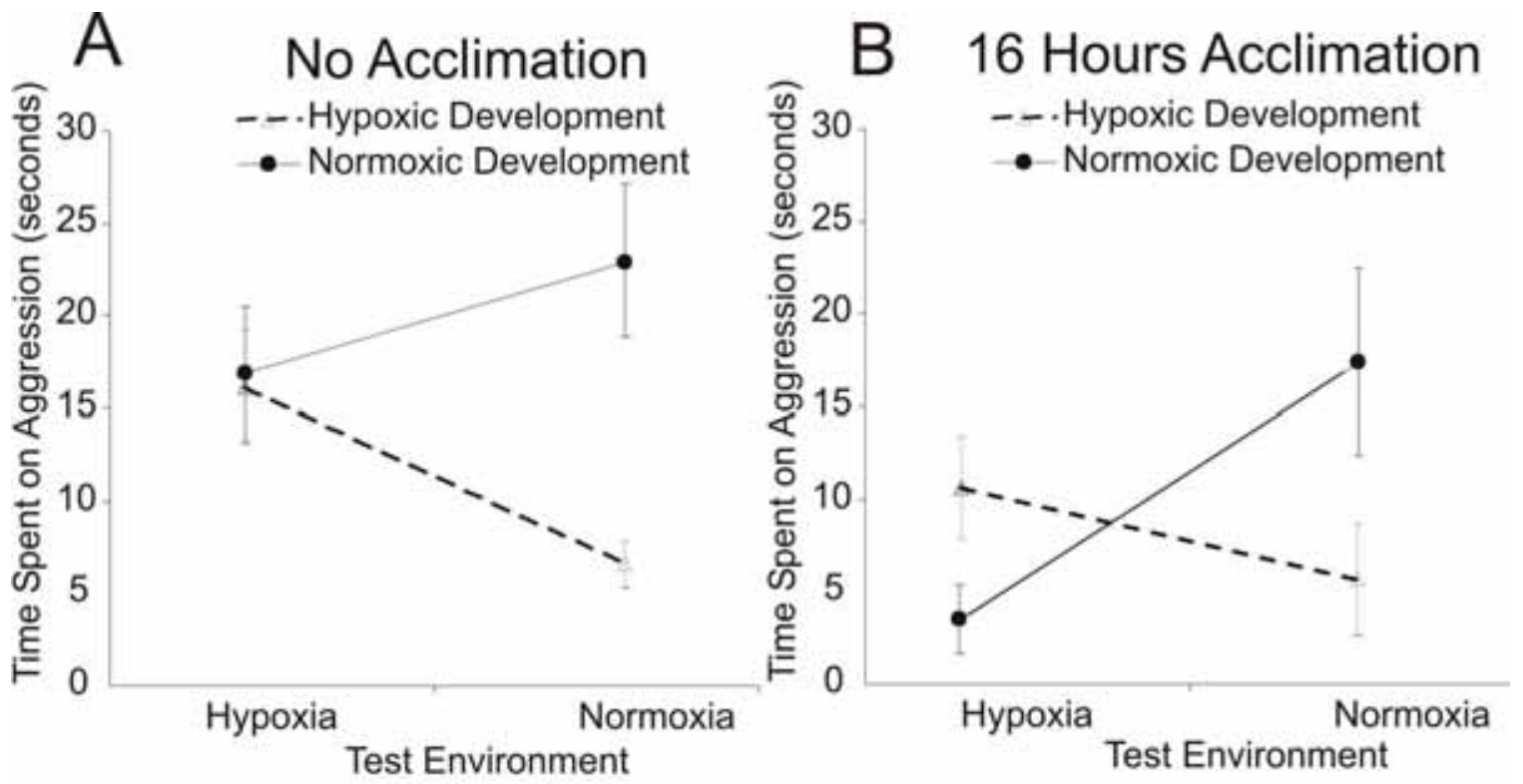

Figure 2: Aggression results. The influence of environment on aggression (A) without acclimation to the environment and (B) with $16 \mathrm{~h}$ of acclimation. Open and solid symbols represent means for hypoxic- and normoxic- reared fish, respectively. Error bars represent standard errors.
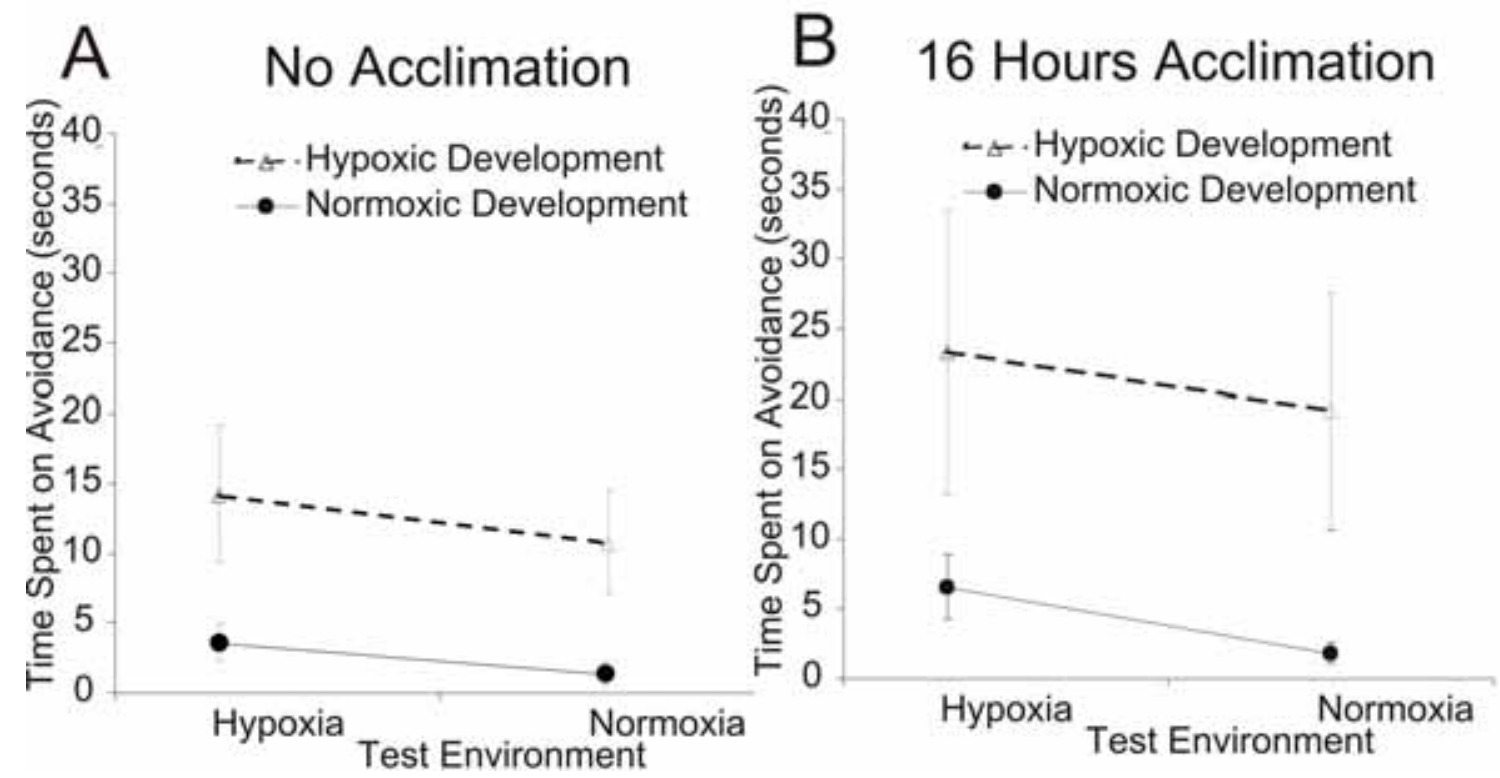

Figure 3: Avoidance results. The influence of environment on avoidance (A) without acclimation to the environment and (B) with $16 \mathrm{~h}$ of acclimation. Open and solid symbols represent means for hypoxic- and normoxia-reared fish, respectively. Error bars represent standard errors. 


\section{BIBLIOGRAPHY}

AGRAWAL, A. A. 2001. Ecology-Phenotypic plasticity in the interactions and evolution of species. Science 294:321-326.

BRENOWITZ, E. A., L. F. BAPTISTA, K. LENT, AND J. C. WINGFIELD. 1998. Seasonal plasticity of the song control system in wild Nuttall's white-crowned sparrows. J. Neurobiol. 34:69-82.

CHAPMAN, L. J., F. GALIS, AND J. SHINN. 2000. Penotypic plasticity and the possible role of genetic assimilation: hypoxia induced trade-offs in the morphological traits of an African cichlid. Ecol. Lett. 3:387-393.

CHEN, D. S., G. VAN DYKHUIZEN, AND W. F. GILLY. 1996. Ontogeny of copepod predation in juvenile squid (Loligo opalescens). Biol. Bull. 190:69-81.

DEWITT, T. J., AND S. M. SCHEINER. 2004a. Phenotypic Plasticity: Functional and Conceptual Approaches. T. J. Dewitt and S. M. Scheiner (eds.). Oxford University Press, Oxford.

DEWITT, T. J., 2004b. Phenotypic variation from single genotypes: a primer, p. 1-9. In: Phenotypic Plasticity: Functional and Conceptual Approaches. T. J. Dewitt and S. M. Scheiner (eds.). Oxford University Press, Oxford.

FALCONER, D. S. 1981. An Introduction to Quantitative Genetics. Longman Group, London.

GRACY, A. Y., J. V. TROLL, AND G. N. SOMERO. 2001. Hypoxia-induced gene expression profiling in the euryoxic fish Gillichthys mirabilis. Proc. Natl. Acad. Sci. USA 98:1993-1998.

NEWMAN, R. A. 1992. Adaptive Plasticity in Amphibian Metamorphosis. Bioscience 42:671-678.

NIKINMAA, M. 2002. Oxygen-dependent cellular functions-why fishes and their aquatic environment are a prime choice of study. Comp. Biochem. Physiol. 133A:1-16. 
PIGLIUCCI, M. 2001. Phenotypic Plasticity: Beyond Nature and Nurture. Johns Hopkins University Press, Baltimore.

ROMBOUGH, P. J. 1988. Respiratory gas exchange, aerobic metabolism, and effects of hypoxia during early life, p. 59-161. In: Fish Physiology. Vol. XI. Part A. W. S. Hoar and D. J. Randall (eds.). Academic Press, San Diego.

ROWLAND, W. 1999. Studying visual cues in fish behavior: a review of ethological techniques. Environ. Biol. Fish. 56:285-305.

SCHEINER, S. M., AND T. J. DEWITT. 2004. Future research directions, p. 201-206. In: Phenotypic Plasticity: Functional and Conceptual Approaches. T. J. Dewitt and S. M. Scheiner (eds.). Oxford University Press, Oxford.

SCHLICHTING, C. D., AND H. SMITH. 2002. Phenotypic plasticity: linking molecular mechanisms with evolutionary outcomes. Evol. Ecol. 16:189-211.

SIH, A. 2004. A behavioral ecological view of phenotypic plasticity, p. 112-125. In: Phenotypic Plasticity: Functional and Conceptual Approaches. T. J. Dewitt and S. M. Scheiner (eds.). Oxford University Press, Oxford.

SNEEDECOR, G. W., AND W. G. COCHRAN. 1989. Statistical Methods. Iowa State University Press, Ames, Iowa.

STEARNS, S. C. 1989. The evolutionary significance of phenotypic plasticity. Bioscience 7:436-445.

TALWAR, P. K., AND A. G. JHINGRAN. 1991. Inland fishes of India and adjacent countries. Vol I. Oxford, New Delhi.

TINBERGEN, N. 1951. The Study of Instinct. Clarendon Press, Oxford.

TON, C., D. STAMATIOU, AND C. C. LIEW. 2003. Gene expression profile of zebrafish exposed to hypoxia during development. Physiol. Genomics 13:97-106.

VIA, S., AND R. LANDE. 1985. Genotype-environment interaction and the evolution of phenotypic plasticity. Evolution 39:505-522.

VIA, S., AND R. LANDE .1987. Evolution of genetic variability in a spatially heterogeneous environment: effects of genotype-environment interaction. Genet. Res. 49:147-156.

WEST-EBERHARD, M. J. 1989. Phenotypic plasticity and the origins of diversity. Ann. Rev. Ecol. Syst. 20:249-278. 
WEST-EBERHARD, M. J. 2003. Developmental Plasticity and Evolution. Oxford University Press, New York.

WHITLOCK, M. C., P. C. PHILLIPS, F. B.-G. MOORE, AND S. J. TONSOR. 1995. Multiple fitness peaks and epistasis. Ann. Rev. Ecol. Syst. 26:601-629. 